\title{
Laboratory to Clinical Investigation of Carbapenem Resistant Acinetobacter baumannii Outbreak in a General Hospital
}

\author{
Mo Guo-xin ${ }^{1}$; She Dan-yang ${ }^{1}$; Guan Xi-zhou ${ }^{1}$; Cui Jun-chang ${ }^{1}$; Wang Rui ${ }^{2}$; Cui Zhi-gang ${ }^{3}$; \\ Chen Liang-an ${ }^{1, *}$ \\ ${ }_{1}^{1}$ Department of Respiratory Disease, Chinese PLA General Hospital, Beijing, China \\ ${ }^{2}$ Institutes of Clinical Pharmacology Chinese, PLA General Hospital, Beijing, Chin \\ ${ }^{3}$ State Key Laboratory for Communicable Diseases, Prevention and Control Institute of Communicable Disease Prevention and Control Chinese Center for Disease Control and \\ Prevention, Beijing, China \\ ${ }^{*}$ Corresponding author: Chen Liang-an, Department of Respiratory Disease of Chinese, PLA General Hospital, Fuxing road 28, P.O.Box: 100853, Beijing, China. Tel: +86-1068295959, Fax: \\ +86-1068295928, E-mail: chenliangan99@163.com
}

Received: June 22, 2013; Revised: October 7, 2013; Accepted: November 18, 2013

\begin{abstract}
Background:The number of reported cases, infected with carbepenem resistant Acinetobacter baumannii(CRAb)and multi-drug resistant (MDR)Acinetobacter species had gradually increased in most PLA general hospital wards from April to June in 2007.

Objectives: We have described the investigation of an outbreak of CRAb and MDR Acinetobacter in PLA general hospital, Beijing. The prospective and retrospective findings were identified and analyzed to study the infection causes.

Materials and Methods: A. baumannii samples were collected from the patients and environment in each hospital unit. The onset times were recorded according to their case information. All samples were characterized by genotype and compared using pulsed-field gel electrophoresis (PFGE). The microorganism susceptibility was tested using the in vitro minimal inhibitory concentration (MIC) breakpoints method.

Results: A total of 69 A. baumannii strains were successfully isolated from 53 patients. About $89.1 \%$ of them were resistant to ampicillin and $89.2 \%$ to cefotaxime and $75.4 \%$ to all standard antibiotics. PFGE analysis revealed that nine of the isolates had unique clones and the epidemic clone types were A, B and C.

Conclusions: The A. baumannii outbreak, was caused by MDRA. baumannii. The strains had widely spread among12 departments especially in surgical intensive care unit (SICU), emergency intensive care unit (EICU) and the department of respiratory disease. The outbreak was more likely caused by the A. baumannii infected or carrier patients and EICU was its origin.
\end{abstract}

Keywords: Acinetobacter baumannii; Drug Resistanc; Sequence Homology; Cross Infection

\section{Background}

The Acinetobacter species have recently attracted clinical and research interests, due to the increase of this genus being reported as a nosocomial pathogen. The Acinetobacter species are subclasses of the Moraxellaceae family which are strictly aerobic, Gram-negative, non-motile, non-lactose-fermenting, oxidase-negative, catalase-positive coccobacilli (1). So far, more than 30 genomic species have been identified in this genus, 17 of which have valid names (2). Acinetobacter baumannii is just one of many Acinetobacter species which can cause disease in humans, but in 2004, the centers for disease control (CDC) of USA reported that $A$. baumannii accounts for approximately $80 \%$ of all reported Acinetobacter infections. A. baumannii can be found in diverse sources such as foods, fresh water and soils. It is also a part of the indigenous flora of healthy human skin, especially at a low density and shortterm duration (3).
A. baumannii is emerging as a cause of numerous global outbreaks which has demonstrated ever-increasing rates of resistances (4-7). Acinetobacter spp. are able to survive for a prolonged time on dry inanimate surfaces; the duration of which has been reported from one $(8,9)$ up to 5 months (10). A. baumannii strains are easily colonized in patients or medical equipment. Specific strains can attach to human epithelial cells through fimbriae or lipopolysaccharide side chains, bind to salivary mucins, or develop biofilm in contact with plastic or glass surfaces. The latter property is of particular clinical affiliation to the catheter-associated infections, (11) being resistance to the antimicrobial agents, and appearing as one of the most important factors of Acinetobacter infections in health-care settings.

\section{Objectives}

From April to June 2007, 53 patients had been infected

Implication for health policy/practice/research/medical education:

A. baumannii has emerged as a significant hospital pathogen, which quickly becomes resistant to the commonly prescribed antimicrobial agents. We reported an outbreak of MDRA. baumannii in a general hospital as well as its identification and chronological transmission pattern.

Copyright (C) 2014,Ahvaz Jundishapur University of Medical Sciences; Published by Kowsar Corp. This is an open-access article distributed under the terms of the Creative Commons Attribution License, which permits unrestricted use, distribution, and reproduction in any medium, provided the original work is properly cited. 
with $A$. baumannii in emergency department and other units of PLA general hospital. We investigated the reason of outbreak, identified the source of the organism and found the suitable controlling methods.

\section{Materials and Methods}

\subsection{Characterizing the Outbreak}

PLAgeneral hospital is a tertiary teaching hospital which offers acute medical and surgical services to thousands of people annually and admits about 80000 patients, 500 of which are accepted in the intensive care unit (ICU). In March 2007, it was found that the A. baumannii infections were very common in different wards of the hospital, while suddenly the number of infected cases increased in a short term with a similar drug resistance pattern were, mostly being drug and carbapenem multiple resistance.

\subsection{Isolation and Identification of the Bacterial Strains}

Written informed consent was obtained from each patient for bacterial isolation as well as clinical information usage. The study was approved by the ethnic review board of the PLA general hospital (ethnic number:301-07-35).

The clinical A. baumannii strains were isolated between April and June 2007 from the respiratory care department, surgical intensive care unit (SICU), emergency intensive care unit and other wards. The environmental strains were collected simultaneously from the SICU regions (including the hands of medical staffs, patients' skin, hospital beds, ventilator tubes and other equipment). The isolated strains were identified by Vitek assay (BiosMerieumx, France) in our microbiology laboratory using standard techniques. In order to guarantee $100 \%$ accuracy of the detected A. baumannii strains, the method of Ribotyping- "PCR amplification for BlaOXA-51-like carbapenemase gene" (12) was applied to identify all A. baumannii strains. Then isolated strains were stored at $-70^{\circ} \mathrm{C}$. At the meantime, the onset times were recorded according to patients' case file.

\subsection{Susceptibility Test}

Minimal inhibitory concentration (MIC) assay was used to determine the drug sensitivity in all isolated strains by agar-dilution method and the corresponding range of drug concentrations were selected according to the recommendation of the national committee for clinical laboratory standards (2007 edition) (13). We used 1:9 ratios to prepare the drug agar panel. Cefoperazone- sulbactam MIC breakpoint was considered as the MIC breakpoint of cefoperazone; moxifloxacin MIC breakpoint was chosen according to the British society antimicrobial chemotherapy (BSAC) guidelines, (14) Tigecycline MIC breakpoint was used according to the FDA recommended value. In our study, isolated strains with intermediate susceptibility or resistance were defined as resistant.

\subsection{Genetic Studies}

Total genomic DNA was extracted from the isolated strains; DNAs were digested overnight by ApaI (Bio Link Inc., USA). A Salmonella serotype braenderup strain (H9812) was chosen as the universal size standard. Restriction fragments were separated on a $1 \%$ gel by pulsedfield gel electrophoresis (PFGE) using the CHEF Mapper® XA system (Bio-Rad Laboratories, USA) in 0.5 X TBE (Trisborate-EDTA) buffer at $8^{\circ} \mathrm{C}$. PFGE was performed with a $6.0 \mathrm{~V} / \mathrm{cm}$ gradient, at $120^{\circ}$ angle, and 7-20-s pulse times for 19 hours. Gel images were taken using the GEL DOC 2000 system (Bio-Rad Laboratories, USA) and analyzed by QUANTITY ONE software.

\subsection{Analysis and Statistical Methods}

The UPGMA (unweighted pair group method with arithmetic mean) method was applied for cluster analysis, using BioNumerics V4.6 software. The relationship between parenteral antibiotic using and carbapenem resistance rates in each unit was assessed by multiple linear regression analysis assays, using SPSS 17.0 software. For categorical variables, the Chi-squared test or Fisher's exact test was used to compare the proportions. Statistical significance was considered as $\mathrm{P} \leq 0.05$.

\section{Results}

\subsection{General Information}

A total of 106 strains were successfully isolated, 86 of them showed positive results in Vitek assay, 72 of which were positive in PCR (Figure 1). After excluding the incomplete data, 69 strains of A.baumannii, isolated from 53 patients during the 3-month period, were successfully identified. Fifteen strains were collected from the respiratory care ward; among them, 14 were isolated from the surgical intensive care unit (SICU); 11 from the emergency intensive care unit; and 29 from the other wards. Another 28 environmental strains were collected simultaneously from the SICU regions (including the hands of medical staffs, patients' skin, hospital beds, ventilator tubes and other equipment). Most of the patients were old (mean age: $56 \pm 21$ years) and the majority of them were male. Nineteen of them died and 34 were alive. The demographic data of these patients is shown in Table 1. 
Guo-xin Met al.

\subsection{Susceptibility Test}

Table 2 shows the results of the susceptibility test. We found that Multi-drug resistance and Carbapenem resistant were the characteristics of the pathogen. The rate of resistance to Carbapenem was 75.4\% (to meropenem was $85.5 \%$ and to Imipenem was $75.4 \%$ ), Among the 19 tested agents, Colistin Sulphate and tigecycline showed high rates of antimicrobial activity against strains, The rate of susceptibility was $100 \%$ and $91.3 \%$ respectively. Moxifloxacin and minocycline had moderate antimicrobial activity against a baumannii, the rate of resistance being $87 \%$ and $36.2 \%$ respectively. Like the other agents, however, cefoperazone-sulbactam, piperacillin-tazobactam and Ticarcillin-clavulanic acid showed a low rate of antimicrobial activity, which the rate of resistance was $88.2 \%, 76.9 \%$ and $82.9 \%$ respectively.

\subsection{Genetic Studies}

The PFGE result (Figure 2) revealed that there were nine unique clones and the most prevalent types were A, B and C. Type A and C have three subtypes, type B has four subtypes and type D to I are individual. The environmental concerned strains are not relevant to the epidemic strains A, B and C. Combined with the in vitro antimicrobial susceptibility test results, we concluded that all type A strains were CRAb, while only a part of type $B$ and $C$ strains were CRAb.

Carbapenem, imipenem and meropenem showed no sensitivity differences to MDR A.baumannii at this outbreak; the moxifloxacin resistance was significantly different in type $C$ strains, compared with type A and B. We chronologically and separately investigated the three clone types (A, B and C), and found out that the type A and $\mathrm{C}$ clones emerged in the emergency department earlier.
Figure 1. PCR Result of 86 Vitek Positive Strains, 72 of which were confirmed to be A. baumannii

\section{A}
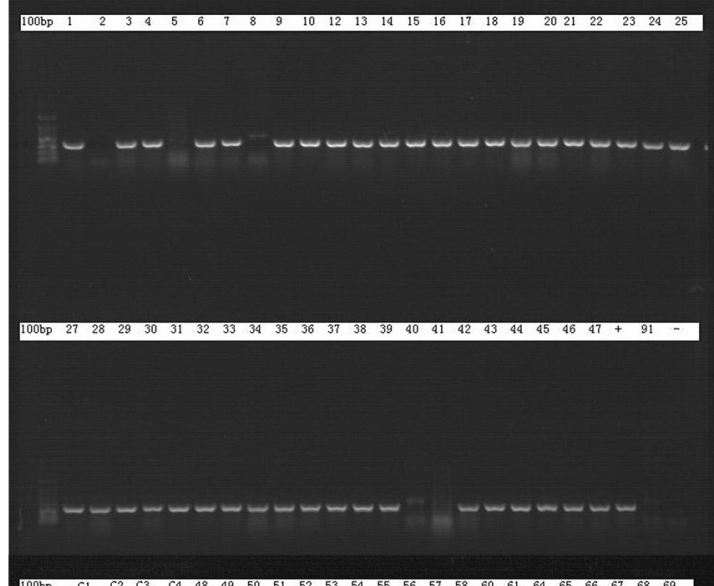

B

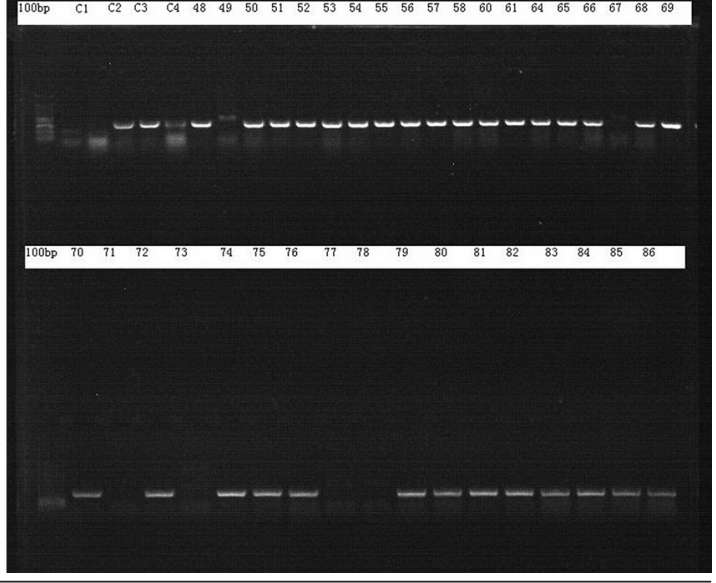

(A) Samples 1-47; (B) samples 48 -86.

\begin{tabular}{ll}
\hline Table 1. Demographic Data of 53 Patients From Whom A. baumannii Strains Were Isolated \\
\hline Features & Variants \\
\hline Total patients, No. & 53 \\
\hline Mean age, Mean \pm SD, $\mathbf{y}$ & $56 \pm 21$ \\
\hline Male patients, No. & 33 \\
\hline Patients in the ICU,No. & 40 \\
\hline Death cases, No. & 19 \\
\hline Average hospitalization, $\mathbf{d}$ & 74 \\
\hline Cephalosporins users before confirmed, No. & 37 \\
\hline p-lactam inhibitors users before confirmed, No. & 38 \\
\hline Operative treatments, No. & 22 \\
\hline Endotracheal intubations, No. & 35 \\
Catheter users, No. & 41 \\
\hline Venous cathezeriation users, No. & 45 \\
\hline Drainage tube users, No. & 22 \\
\hline
\end{tabular}


Guo-xin Met al.

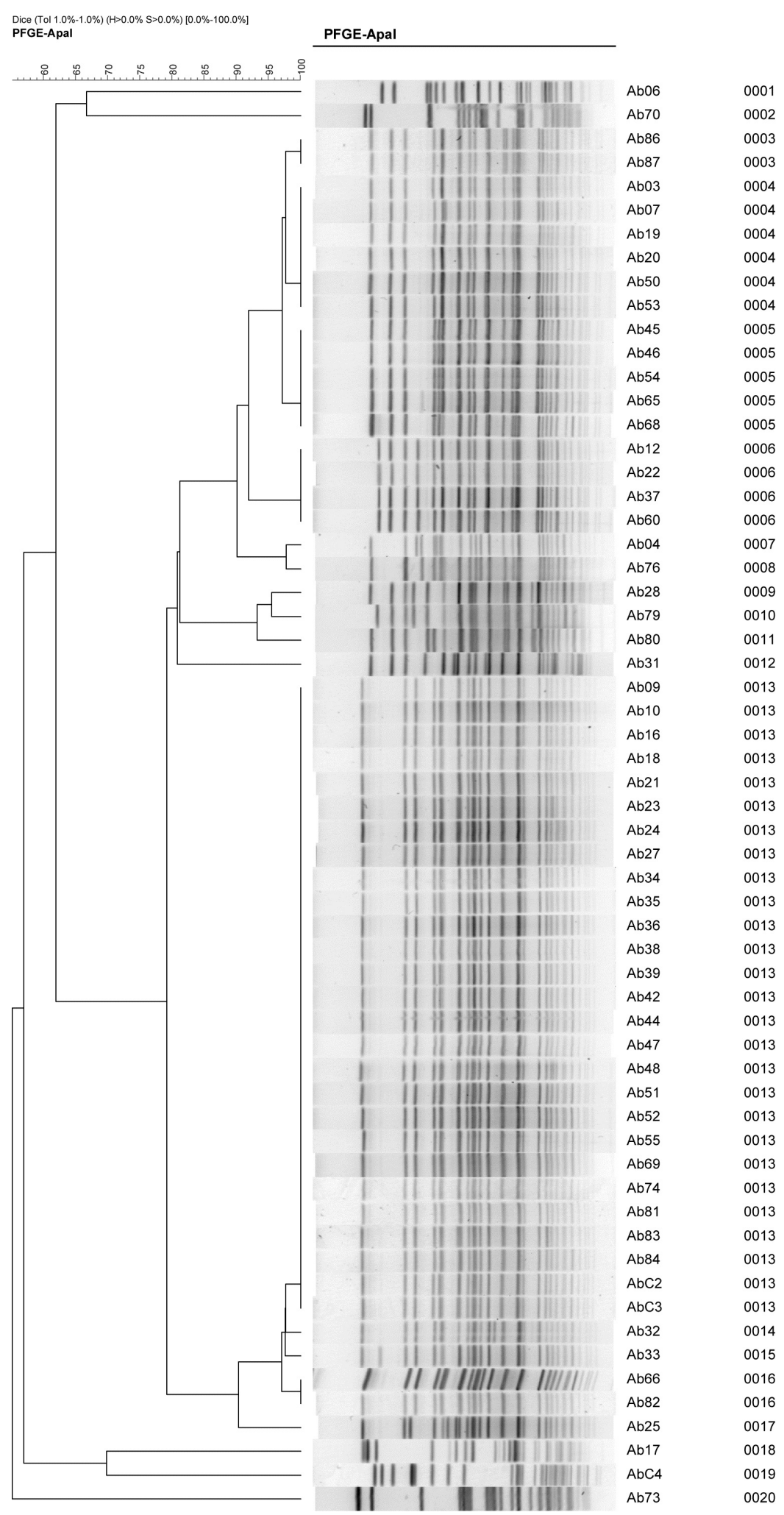

Figure 2. The PFGE Clustering Analysis Results of 62 A. baumannii Strains 
Guo-xin Met al.

Table 2. The MIC and Drug Resistance Rates of A. baumannii Strains to Antimicrobial Agents

\begin{tabular}{|c|c|c|c|c|}
\hline Antibiotic & MIC $^{\mathrm{C}}$ Range (mg/mL) & $\mathrm{MIC}^{50 \mathrm{c}}$ & MIC $^{90 \mathrm{c}}$ & Drug Resistance Rate \\
\hline Ampicillin & $16->512$ & $>512$ & $>512$ & 89.9 \\
\hline Ampicillin-Sulbactam & $1-512$ & 32 & 64 & 82.9 \\
\hline Piperacillin-Tazobactam & $8->512$ & 256 & 512 & 76.9 \\
\hline Ticarcillin-Clavulanic acid & $0.25<->512$ & $>512$ & $>512$ & 82.9 \\
\hline Ceftazidime & $2->512$ & 128 & 256 & 91.3 \\
\hline Cefepime & $1-256$ & 64 & 64 & 80 \\
\hline Cefotaxime & $8->512$ & 512 & 512 & 89.8 \\
\hline Cefoperazone & $8->512$ & $>512$ & $>512$ & 95.7 \\
\hline Cefoperazone-Sulbactam & $2-256$ & 64 & 64 & 88.2 \\
\hline Imipenem & $0.125-128$ & 32 & 64 & 75.4 \\
\hline Meropenem & $0.25-128$ & 32 & 64 & 85.5 \\
\hline Polymyxin B sulfate & $0.5-2$ & 1 & 1 & 0 \\
\hline Gentamicin & $0.5->256$ & $>256$ & $>256$ & 87 \\
\hline Amikacin & $2->512$ & $>512$ & $>512$ & 84 \\
\hline Minocycline & $0.25-32$ & 8 & 32 & 36.2 \\
\hline Tigecycline $^{a}$ & $0.125-8$ & 1 & 2 & 8.7 \\
\hline Levofloxacin & $0.125-64$ & 8 & 32 & 84 \\
\hline Moxifloxacin $^{b}$ & $0.06-64$ & 8 & 32 & 87 \\
\hline Trimethoprim-Mulphamethoxazole & $0.025->32$ & $>32$ & $>32$ & 85.5 \\
\hline
\end{tabular}

a Tigecycline was referenced according to the CLSI Enterobacteriaceae information.

b Empirical clinical drug usage, referenced break point of BSAC (British society antimicrobial chemotherapy).

C MIC, Minimum inhibitory concentration; MIC ${ }^{50}$, Minimum Inhibitory Concentration required to inhibit the growth of $50 \%$ of organisms; MIC 90 , Minimum Inhibitory Concentration required to inhibit the growth of $90 \%$ of organisms

\section{Discussion}

A. baumannii is widely spread in the nature, which could be collected from soil, water, (15) humidifiers, pillows, ventilators, medical staff skins and surface of other items (16-18). Its survival at the surfaces has made it the hospital infection pathogen (19). In our study, the frequency of the collected positive A. baumannii samples was $21.7 \%$ in the respiratory care medicine department, $20.3 \%$ in the intensive care unit and $15.9 \%$ in the emergency care unit. Generally, mechanical ventilation is often considered as the most important $A$. baumannii pneumonia-causing factor (19-23). Application of antibiotics in patients with severe infections increases the opportunity of antimicrobial selective pressure accordingly (24). Correspondingly, the number of infections caused by multi-drug resistant $A$. baumannii strains are increasing. Current reports showed that only a minority of antibacterial drugs such as polymyxin, tigecycline and formulations containing Sulbactam can be used for the treatment of A. baumannii (25-27).

In this study, the isolated strains had similar resistance characteristics, the CRAb rate was $75.4 \%$, making it the main cause of the A. baumannii outbreak. The PFGE analysis resultrevealed the preliminary answer of the out- break: MDR A. baumannii occurred in 12 medical rooms of seven wards located in three medical buildings, the A1 type of A. baumannii was isolated from the hands of nursing staffs as well as the patients' body fluids; meanwhile, isolated samples from nurses' washed hands were negative. These results demonstrate that the CRAb on the hands of care staffs was the important source of pathogenic Acinetobacter infections in the hospital. Secondly, we analyzed the transmission pattern of MDR A. baumannii in three major departments-the respiratory care, emergency and the SICU department, which showed that most of the patients in the respiratory care department were only staying in that specific ward, not having much contact with others; Emergency department had complex patients and diseases with the most transferring frequency, patients in SICU generally had serious conditions and more transferring thus the transfer of patients possessing MDR A. baumannii infection was the main reason of the spread between the units.

Accordingly, we investigated the patients with MDR A. baumannii infection carefully and found the main popular type in the respiratory care ward, which was type A and not C. Only one isolated B2 type (No. 68) was transferred from the SICU. In the SICU, A and B types were the 
epidemic strains and only one type C specimen (No. 79) was confirmed to be transferred from the emergency ICU. Emergency department had all types A, B and C epidemic strains. According to the medical records analysis results, emergency rooms have the highest number of epidemic strains, thus we deduced the possible pattern of MDR A. baumannii transmission outbreak in the hospital: emergency department has significant possibility as the pathogen occurrence location, due to the high number of patients' transfers in the emergency and SICU departments compared with the others.

Patients with a clinical A. baumannii infection have to pay additional charges because they have to stay in the hospital for an average of 13 days longer than a patient without A. baumannii infection (28). A. baumannii may not be particularly virulent, but it can cause unnecessary diseases and impose extra expenses on critically ill patients, thus the transmission of such pathogen should be limited.

In conclusion, A. baumannii infections are associated with considerable morbidity and mortality. Therefore, proceedings such as strengthening the concept of hand washing in clinical staffs as well as strengthening the isolation of patients with MDRAb infection, and effective anti-infection treatments for patients could effectively prevent the spread of A. baumannii.

\section{Acknowledgements}

There is no acknowledgement.

\section{Authors' Contribution}

MO Guo-xin and CHEN Liang-an defined the research theme. SHE Dan-yang and GUAN Xi-zhou carried out the laboratory experiments. CUI Jun-chang and WANG Rui interpreted the data. CUI Zhi-gang co-designed the dispersal and colonization experiments and associated the data collection.

\section{Financial Disclosure}

The authors declare there is no financial interest to disclose.

\section{Funding/Support}

The authors had no funding for the study.

\section{References}

1. Karageorgopoulos Drosos E, Falagas Matthew E. Current control and treatment of multidrug-resistant Acinetobacter baumannii infections. Lancet Infect Dis. 2008;8(12):751-762.

2. Chan JZ, Halachev MR, Loman NJ, Constantinidou C, Pallen MJ. Defining bacterial species in the genomic era: insights from the genus Acinetobacter. BMC Microbiol. 2012;12:302.

3. Cetin ES, Durmaz R, Tetik T, Otlu B, Kaya S, Caliskan A. Epidemiologic characterization of nosocomial Acinetobacter baumanni infections in a Turkish university hospital by pulsed-field gel electrophoresis. Am JInfect Control. 2009;37(1):56-64.
4. Amudhan SM, Sekar U, Arunagiri K, Sekar B. OXA beta-lactamasemediated carbapenem resistance in Acinetobacter baumannii. Indian JMed Microbiol. 2011;29(3):269-74.

5. Jeong SH, Bae IK, Park KO, An YJ, Sohn SG, Jang SJ, et al. Outbreaks of imipenem-resistant Acinetobacter baumannii producing carbapenemases in Korea.J Microbiol. 2006;44(4):423-31.

6. Urban C, Segal-Maurer S, Rahal JJ. Considerations in control and treatment of nosocomial infections due to multidrug-resistant Acinetobacter baumannii. Clin Infect Dis. 2003;36(10):1268-74.

7. Valenzuela JK, Thomas L, Partridge SR, van der Reijden T, Dijkshoorn L, Iredell J. Horizontal gene transfer in a polyclonal outbreak of carbapenem-resistant Acinetobacter baumannii. J Clin Microbiol. 2007;45(2):453-60.

8. Hirai Y. Survival of bacteria under dry conditions; from a viewpoint of nosocomial infection. J Hosp Infect. 1991;19(3):191-200.

9. Livermore DM, Hill RL, Thomson H, Charlett A, Turton JF, Pike R, et al. Antimicrobial treatment and clinical outcome for infections with carbapenem- and multiply-resistant Acinetobacter baumannii around London. Int J Antimicrob Agents. 2010;35(1):19-24.

10. Webster C, Towner KJ, Humphreys H. Survival of Acinetobacter on three clinically related inanimate surfaces. Infect Control Hosp Epidemiol. 2000;21(4):246.

11. Seifert H, Dijkshoorn L, Gerner-Smidt P, Pelzer N, Tjernberg I, Vaneechoutte M. Distribution of Acinetobacter species on human skin: comparison of phenotypic and genotypic identification methods. J Clin Microbiol. 1997;35(11):2819-25.

12. Turton JF, Woodford N, Glover J, Yarde S, Kaufmann ME, Pitt TL Identification of Acinetobacter baumannii by detection of the blaOXA-51-like carbapenemase gene intrinsic to this species. $J$ Clin Microbiol. 2006;44(8):2974-6.

13. Ocana V, Silva C, Nader-Macias ME. Antibiotic susceptibility of potentially probiotic vaginal lactobacilli. Infect Dis Obstet Gynecol. 2006;2006:18182.

14. Uete G, Matsuo K, Uete T. [Clinical laboratory approach to evaluate efficacy of amikacin. Reevaluation of in vitro MIC break points in disc susceptibility test]. Jpn J Antibiot. 1992;45(5):489501

15. Rocha LA, Martin MJ, Pita S, Paz J, Seco C, Margusino L, et al. Prevention of nosocomial infection in critically ill patients by selective decontamination of the digestive tract. A randomized, double blind, placebo-controlled study. Intensive Care Med. 1992;18(7):398-404.

16. Cefai C, Richards J, Gould FK, McPeake P. An outbreak of Acinetobacter respiratory tract infection resulting from incomplete disinfection of ventilatory equipment. J Hosp Infect. 1990;15(2):177182.

17. Sherertz RJ, Sullivan ML. An Outbreak of Infections with Acinetobacter calcoaceticus in Burn Patients: Contamination of Patients' Mattresses. J Infect Dis. 1985;151(2):252-258.

18. Weernink A, Severin WPJ, Tjernberg I, Dijkshoorn L. Pillows, an unexpected source of Acinetobacter.J Hosp Infect. 1995;29(3):189199.

19. Husni RN, Goldstein LS, Arroliga AC, Hall GS, Fatica C, Stoller JK, et al. Risk factors for an outbreak of multi-drug-resistant Acinetobacter nosocomial pneumonia among intubated patients. Chest. 1999;115(5):1378-82.

20. Bergogne-Berezin E, Towner KJ. Acinetobacter spp. as nosocomial pathogens: microbiological, clinical, and epidemiological features. Clin Microbiol Rev. 1996;9(2):148-65.

21. Pallares R, Gudiol F, Linares J, Ariza J, Rufi G, Murgui L, et al. Risk factors and response to antibiotic therapy in adults with bacteremic pneumonia caused by penicillin-resistant pneumococci. N Engl J Med.1987;317(1):18-22.

22. Rello J, Ausina V, Ricart M, Puzo C, Quintana E, Net A, et al. Risk factors for infection by Pseudomonas aeruginosa in patients with ventilator-associated pneumonia. Intensive Care Med 1994;20(3):193-8

23. Zimmerman S, Gruber-Baldini AL, Hebel JR, Sloane PD, Magaziner J. Nursing home facility risk factors for infection and hospitalization: importance of registered nurse turnover, administration, and social factors. JAm Geriatr Soc. 2002;50(12):1987-95.

24. Torres A, Aznar R, Gatell JM, Jimenez P, Gonzalez J, Ferrer A, et al. 
Guo-xin Met al.

Incidence, risk, and prognosis factors of nosocomial pneumonia in mechanically ventilated patients. Am Rev Respir Dis. 1990;142(3):523-8.

25. Falagas ME, Kasiakou SK. Colistin: the revival of polymyxins for the management of multidrug-resistant gram-negative bacterial infections. Clin Infect Dis. 2005;40(9):1333-41.

26. Hsueh PR, Teng LJ, Chen CY, Chen WH, Yu CJ, Ho SW, et al. Pandrug-resistant Acinetobacter baumannii causing nosocomial infections in a university hospital, Taiwan. Emerg Infect Dis.
$2002 ; 8(8): 827-32$.

27. Navon-Venezia S, Leavitt A, Carmeli Y. High tigecycline resistance in multidrug-resistant Acinetobacter baumannii. J Antimicrob Chemother. 2007;59(4):772-4.

28. Young LS, Sabel AL, Price CS. Epidemiologic, clinical, and economic evaluation of an outbreak of clonal multidrugresistant Acinetobacter baumannii infection in a surgical intensive care unit. Infect Control Hosp Epidemiol. 2007;28(11):1247-54. 